\title{
Genome-Wide Identification and Analysis of the AP2 Transcription Factor Gene Family in Wheat (Triticum aestivum L.)
}

\author{
Yue Zhao ${ }^{1}$, Renyi $\mathrm{Ma}^{1}$, Dongliang X ${ }^{1}$, Huihui $\mathrm{Bi}^{2 *}$, Zongliang Xia ${ }^{1 *}$ and Huiru Peng ${ }^{3}$ \\ ${ }^{1}$ College of Life Science, Henan Agricultural University, Zhengzhou, China, ${ }^{2}$ College of Agronomy/Collaborative Innovation \\ Center of Henan Grain Crops/National Key Laboratory of Wheat and Maize Crop Science, Henan Agricultural University, \\ Zhengzhou, China, ${ }^{3}$ State Key Laboratory for Agrobiotechnology, Key Laboratory of Crop Heterosis and Utilization (MOE), \\ Beijing Key Laboratory of Crop Genetic Improvement, China Agricultural University, Beijing, China
}

OPEN ACCESS

Edited by: Kimberley Cathryn Snowden, The New Zealand Institute for Plant \& Food Research Ltd, New Zealand

Reviewed by: Hester Sheehan, University of Cambridge, United Kingdom Jinhui Chen,

Nanjing Forestry University, China

${ }^{*}$ Correspondence: Huihui Bi

huihui.bi@henau.edu.cn Zongliang Xia xiaz108@163.com

Specialty section: This article was submitted to Plant Development and EvoDevo, a section of the journal

Frontiers in Plant Science

Received: 23 July 2019 Accepted: 13 September 2019 Published: 11 October 2019

Citation:

Zhao Y, Ma R, Xu D, Bi H, Xia Z and Peng $H$ (2019) Genome-Wide Identification and Analysis of the AP2 Transcription Factor Gene Family in Wheat (Triticum aestivum L.).

Front. Plant Sci. 10:1286. doi: 10.3389/fpls.2019.01286
The AP2 transcription factors play important roles in regulating plant growth and development. However, limited data are available on the contributions of AP2 transcription factors in wheat (Triticum aestivum L.). In the present study, a total of 62 AP2 genes were identified in wheat from a genome-wide search against the latest wheat genome data. Phylogenetic and sequence alignment analyses divided the wheat AP2 genes into 3 clusters, euAP2, euANT, and basalANT. Chromosomal distribution, gene structure and duplication, and motif composition were subsequently investigated. The 62 TaAP2 genes were unevenly distributed on 21 chromosomes. Twenty-four homologous gene sets among $\mathrm{A}, \mathrm{B}$, and $\mathrm{D}$ sub-genomes were detected, which contributed to the expansion of the wheat AP2 gene family. The expression levels of TaAP2 genes were examined using the WheatExp database; most detected genes exhibited tissue-specific expression patterns. The transcript levels of 9 randomly selected TaAP2 genes were validated through qPCR analyses. Overexpression of TaAP2-10-5D, the most likely homolog of Arabidopsis ANT gene, increased organ sizes in Arabidopsis. Our results extend our knowledge of the AP2 gene family in wheat, and contribute to further functional characterization of AP2s during wheat development with the ultimate goal of improving crop production.

Keywords: AP2, Arabidopsis, expression pattern, organ size, phylogenetic tree, transgenic, wheat

\section{INTRODUCTION}

The APETALA2/Ethylene-Responsive Factor (AP2/ERF) superfamily is one of the largest groups of transcription factors (TFs) in plants. The AP2/ERF superfamily is defined by the conserved AP2 DNA binding domains of 57-66 amino acids (Okamuro et al., 1997). In general, the AP2/ERF superfamily is divided into three separate families, which are AP2, ERF, and RAV families, based on the number of AP2 domains and sequence similarities. The AP2 family proteins contain two repeated AP2 domains; the ERF family proteins contain a single AP2 domain; the RAV (related to ABI3/VP1) family proteins contain two different DNA binding domains, AP2 and B3 (Sakuma et al., 2002; Nakano et al., 2006). The AP2 family was subdivided into the euAP2, eu-AINTEGUMENTA (euANT) and basalANT groups according to the amino acid sequence of the double AP2 domain 
and the nuclear localization sequence (Shigyo et al., 2006; Wang et al., 2016a; Dipp-Alvarez and Cruz-Ramirez, 2019).

The AP2 family genes play key roles in the reproductive and vegetative organs development (Gil-Humanes et al., 2009; Wang et al., 2016a; Scheres and Krizek, 2018). In Arabidopsis, 18 AP2 family genes have been identified (Sakuma et al., 2002). Arabidopsis AP2 is the most well-studied gene in the AP2 family. AtAP2 gene plays a central role in the specification of floral organ identities (Bowman et al., 1989; Kunst et al., 1989), the regulation of flowering time and floral meristem (Coen and Meyerowitz, 1991), the control of floral homeotic gene expression (Drews et al., 1991; Mandel et al., 1992), and the modulation of seed development (Jofuku et al., 2005) in Arabidopsis. The TARGETS OF EAT (TOE) genes, homologs of $A t A P 2$, were shown to affect flowering time in Arabidopsis by repressing the expression of flowering regulatory genes (Mathieu et al., 2009; Yant et al., 2010). The TOE genes include TOE1, TOE2, TOE3, SCHLAFMÜTZE (SMZ), and SCHNARCHZAPFEN (SNZ); these genes are negatively regulated by microRNA172 (miR172) at the posttranscriptional level (Aukerman and Sakai, 2003; Chen, 2004; Zhang et al., 2015b). In addition, the ANT, ANT-LIKE5 (AIL5), AIL6, and AIL7 genes, members of the euANT group of the AP2 family, regulate ovule development and floral organ growth (Elliott et al., 1996; Klucher et al., 1996; Nole-Wilson et al., 2005; Krizek, 2009; Krizek, 2015). Another members of the euANT group, $A t B B M$ and $P L T$ genes, were reported to likely function during embryogenesis (Boutilier et al., 2002), and function in roots (Aida et al., 2004), respectively. In rice (Oryza sativa L.), 23 AP2 members have been identified (Rashid et al., 2012), and some of them have been functionally characterized, including SMALL ORGAN SIZE1 (SMOS1) (Aya et al., 2014), SUPERNUMERARY BRACT (SNB) and INDETERMINATE SPIKELET1 (OsIDS1) (Lee and An, 2012; Lee et al., 2014; Jiang et al., 2019). SNB and OsIDS1 together play important roles in inflorescence architecture and the establishment of floral meristems (Lee and An, 2012). Recently, it was found that SNB controls rice seed shattering and seed size (Jiang et al., 2019). Maize IDS1 is expressed in roots, leaves, inflorescence primordia, spikelet pair primordia, and embryos (Chuck et al., 2008). In the ids1 mutant, the spikelet meristem becomes indeterminate and produces additional florets (Chuck et al., 2008). It was also reported that maize AP2-like gene glossy15 regulates leaf epidermal cell identity (Moose and Sisco, 1996) and kernel row number 1 (krn1) enhanced kernel row numbers (Wang et al., 2019a).

Wheat (Triticum aestivum $\mathrm{L} ., 2 \mathrm{n}=6 \mathrm{x}=42$, BBAADD) is one of the most important crops in the world. Extensive research has been conducted on the AP2/ERF superfamily transcription factors in wheat, but attention has mainly focused on the ERF family because of their significant roles in abiotic and biotic stresses (Xu et al., 2011; Kulkarni et al., 2017). Up to now, only three AP2 family genes ( $Q, T a A P 2$, and TaPARG) have been characterized in wheat. Gene $Q$ has pleiotropic influences on many traits, such as grain and spike morphology, plant height, and spike emergence time (Simons et al., 2006; Greenwood et al., 2017; Liu et al., 2018; Xie et al., 2018; Xu et al., 2018). $T a A P 2$, the gene underlying lodicule development, is required for engineering of cleistogamous wheat (Ning et al., 2013). Li et al. (2016) reported that TaPARGs play a key role in growth and development, including regulation of plant architecturerelated traits and yield-related traits. To further explore the roles of AP2 genes in wheat, we performed a genome-wide search of AP2 family genes using the currently released wheat genome data. Phylogenetic, chromosomal location, gene structure, and expression pattern analyses of the identified wheat AP2 genes were undertaken. One of the family members designated TaAP210 was demonstrated to play an important role in regulating organ size. These results contribute to enrich our knowledge of AP2 gene family in wheat, and lay a basis for future functional analysis of the wheat AP2 family genes.

\section{MATERIALS AND METHODS}

\section{Database Search and Physical Locations of AP2s in Wheat}

The wheat genome sequencing has been completed in 2018 (Appels et al., 2018), and filtered protein and coding sequences have also become available ${ }^{1}$. First, all the wheat AP2 domaincontaining protein sequences were downloaded from the Ensembl Plants database ${ }^{2}$ after a hidden Markov model (HMM) search using the HMM profiles of the AP2 domain (Pfam ID: PF00847) as queries. Then, the sequences were checked by $\mathrm{CDD}^{3}$ (Marchler-Bauer et al., 2017) to confirm the presence of the AP2 domain. Furthermore, all candidate sequences were analyzed by means of SMART ${ }^{4}$ (Letunic et al., 2015) and PFAM databases ${ }^{5}$ to eliminate the sequences not containing two AP2 domains. As a final quality check, the existence of all candidate sequences were examined by BLASTN similarity search against the wheat ESTs deposited in NCBI database ${ }^{6}$. The isoelectric points $(p I)$ and protein molecular weights (MW) of the putative TaAP2s were calculated using the ExPASy online tool ${ }^{7}$. The physical locations of all designated AP2 genes were obtained from the URGI IWGSC BLAST ${ }^{8}$. The chromosomal location image was generated by the MapInspect software ${ }^{9}$. Arabidopsis AP2 sequences were downloaded from The Arabidopsis Information Resources $\left(\mathrm{TAIR}^{10}\right.$ ) and rice AP2 sequences were from Rice Genome Annotation Project (RGAP ${ }^{11}$ ) (Kawahara et al., 2013).

\section{Gene Structure, Multiple Sequence Alignment, and Construction of Phylogenetic Tree}

The exon/intron structures were constructed by GSDS ${ }^{12}$ (Hu et al., 2015) using the coding sequences (CDS) and

\footnotetext{
${ }^{1}$ http://www.wheatgenome.org/

2 http://plants.ensembl.org/index.html

${ }^{3}$ https://www.ncbi.nlm.nih.gov/Structure/cdd/wrpsb.cgi

${ }^{4} \mathrm{http}: / /$ smart.embl-heidelberg.de/

${ }^{5}$ http://pfam.xfam.org/

${ }^{6}$ https://blast.ncbi.nlm.nih.gov/Blast.cgi

${ }^{7}$ http://web.expasy.org/compute_pi/

${ }^{8}$ https://urgi.versailles.inra.fr/blast_iwgsc/blast.php

${ }^{9} \mathrm{http}: / /$ www.plantbreeding.wur.nl/UK/software_mapinspect.html

${ }^{10} \mathrm{http}: / /$ www.arabidopsis.org/

${ }^{11} \mathrm{http}: / /$ rice.plantbiology.msu.edu/

${ }^{12}$ http://gsds.cbi.pku.edu.cn/
} 
corresponding genomic sequences retrieved from the Ensembl Plants database ${ }^{2}$. Multi-sequence alignments were carried out using the ClustalW version 2.0 with default settings (Larkin et al., 2007). Phylogenetic and molecular evolutionary analysis was conducted by MEGA version 6 using the Neighbor-Joining and Maximum-Likelihood methods (Tamura et al., 2013). The reliability of phylogenetic trees was tested using bootstrapping with 1,000 replicates.

\section{Analysis of Gene Duplication}

To identify duplicated gene pairs, we defined gene duplication according to the following criteria (Wang et al., 2016b): (1) the alignable nucleotide sequence covered was $>80 \%$ of the longer aligned gene, and (2) the aligned region had an identity $>80 \%$.

\section{Motif Analysis}

MEME $^{13}$ (Multiple Em for Motif Elicitation, version 4.11.4) was used to identify conserved motifs within complete AP2 protein sequences of wheat using the following parameters: optimum motif width set to $\geq 6$ and $\leq 50$; maximum number of motifs: 16 .

\section{Analysis of Gene Expression Profiles}

Gene expression data of TaAP2 genes in different wheat tissues (spike, root, leaf, grain, and stem) of the hexaploid bread wheat (var. Chinese Spring) were obtained from the WheatExp database ${ }^{14}$ (Pearce et al., 2015). The tissues include leaves at seedling and reproductive stages, roots at seedling and reproductive stages, young spikes, spikes at flowering, stems at anthesis stage, and 14 days after anthesis (DAA) grains. The expression patterns were presented as a heat map, which reflected the FPKM (Fragments Per Kilobase of transcript per Million mapped reads), with red indicating high expression levels, yellow indicating medium expression levels, and blue indicating almost no expression. The heat map was plotted using the Heatmap Illustrator HemI v.1.0 (Deng et al., 2014).

\section{Plant Materials and Growth Conditions}

Seeds of var. Chinese Spring were grown under controlled glasshouse conditions. Roots, stems, leaves, developing spikes (length: $10-20 \mathrm{~mm}$ ), and spikes at flowering were collected from three wheat plants, and all of the samples were immediately frozen in liquid nitrogen and then stored at $-80^{\circ} \mathrm{C}$ for RNA extraction.

The Arabidopsis Col-0 was used to generate TaAP2-10-5D transgenic plants. The Arabidopsis plants were grown under controlled conditions in a greenhouse at $22^{\circ} \mathrm{C}$ with $60 \%$ relative humidity and the photoperiod of $16 \mathrm{~h}$ light and $8 \mathrm{~h}$ dark at $110 \mu \mathrm{mol} \mathrm{m} \mathrm{m}^{-2} \mathrm{~s}^{-1}$ or on half-strength Murashige and Skoog (MS) medium. After grown for 4 weeks on soil, images of each plant were taken from the above, and used for rosette diameter measurements using ImageJ software ${ }^{15}$. Plant height and fresh weight were recorded at the mature period. The silique length was measured from the three longest siliques of each plant. A total of 10-20 plants per genotype were analyzed in each experiment.

\footnotetext{
${ }^{13} \mathrm{http}: / /$ meme-suite.org/tools/meme

${ }^{14} \mathrm{https} / / /$ wheat.pw.usda.gov/WheatExp/

${ }^{15}$ http://imagej.nih.gov/ij/
}

\section{RNA Extraction and qPCR Analysis}

Total RNA was extracted using TRIzol reagent (Invitrogen, USA). The isolated RNA (2 $\mu \mathrm{g})$ was treated with DNase I (TaKaRa, Japan), and cDNA synthesis was conducted using the 5X All-In-One RT MasterMix (abm, Canada) according to the manufacturer's protocol. Real-time quantitative PCR (qPCR) was performed on StepOne ${ }^{\mathrm{TM}}$ and StepOnePlus ${ }^{\mathrm{TM}}$ RealTime PCR Systems (Life Technologies, USA) using the SYBR ${ }^{\circledR}$ Green reaction kit (TaKaRa, Japan). The wheat $\beta$-actin and AtActin2 were used as internal reference genes in wheat and Arabidopsis, respectively. The conditions for qPCR have been described previously (Zhao et al., 2018). Three biological replicates and three technical replicates were applied for all qPCR analyses in this study. The relative gene expression levels were calculated using the $2^{-\Delta \mathrm{Ct}}$ method (Livak and Schmittgen, 2001). The primers used for the qPCR analysis were listed in Table S1.

\section{Generation of Transgenic Plants}

The TaAP2-10-5D CDS was amplified through PCR using primers TaAP2-10-tDF/R, and subsequently subcloned into the pSuper1300 plant expression vector harbouring the mannopine synthase (mas) promoter. The transformation of the obtained recombinant vector into wild type (Col-0) Arabidopsis was performed via Agrobacterium tumefaciens (strain GV3101) mediated floral-dip method (Clough and Bent, 1998). The transgenic plants were screened by $0.1 \%$ Hygromycin B solution and then confirmed by qPCR.

\section{Microscopy Counts of Leaf Epidermal Cells}

The fifth leaf was collected from greenhouse-grown 4-weekold wheat plants $(n=5)$ for counts of leaf epidermal cells, as described previously (Zhao et al., 2017). Briefly, chlorophyll was removed by washing the leaf $2-3$ times until the washing solution (70\% ethanol) remained clear. The leaf epidermis was peeled off and mounted on a glass slide under a cover glass. Images were taken using a Nikon Ti-U microscope. All whole cells captured in the view field were counted.

\section{RESULTS}

\section{Identification of AP2 Gene Family in Wheat}

To identify wheat AP2 genes, a HMM search was conducted using the HMM profiles of the AP2 domain (Pfam ID: PF00847) as queries against the latest genome data of wheat in the Ensembl Plants database. A total of 565 sequences were discovered as potentially encoding AP2 domain(s)-containing proteins in the wheat genome. Subsequently, all putative genes were examined to check the number of AP2 domains in the encoded proteins using the SMART program. We found that the protein sequences of 62 genes contain two AP2 domains, while the remaining 503 sequences contain a single or partial AP2 domain. Detailed information on the 62 AP2 genes was listed in Table S2. The lengths of TaAP2 proteins ranged from 328 (TaAP2-18-4D) to 703 amino acids (TaAP2-3-3D). The isoelectric points $(p \mathrm{I})$ ranged from 5.16 (TaAP2-19-6D) to 9.47 (TaAP2-15-6A). 


\section{Classification and Phylogenetic Analyses of TaAP2s}

In order to precisely reveal the evolutionary relationships of the TaAP2 proteins, we performed phylogenetic analyses of 103 AP2 proteins, including 62 wheat, 18 Arabidopsis and 23 rice AP2s using both neighbor-joining method (Figure 1) and maximumlikelihood algorithm (Figure S1). The genes from different wheat sub-genomes but in adjacent branches of phylogenetic trees were regarded as different copies of each member of the TaAP2 gene family. Thus, we obtained 24 wheat AP2 members designated from TaAP2-1 to TaAP2-24, and the homologous copies of each member were distinguished by subjoining the wheat sub-genome symbols A, B or D. According to the previous classification of AP2 genes in Arabidopsis and rice (Kim et al., 2006; Shigyo et al., 2006), wheat TaAP2 genes were also categorized as three types: euAP2, euANT, and basalANT. The bootstrap values are reasonably high for the derived subclades and for the euAP2 group; however, these support values are lower at more basal branches. For instance, the support value for one branch of basal ANT group and the euANT group is $45 \%$ (Figure 1). Low support values were also observed in the ANT groups in the ML tree (Figure S1). Therefore, it is difficult to differentiate the basalANT group from the euANT group based on phylogenetics. Next, we conducted a sequence alignment of all the 24 members of wheat AP2 proteins. As expected, all the wheat proteins in the putative euANT group contain euANT1-4 motifs (Figure S2), which are exclusively present in the euANT group in plants (Kim et al., 2006). Finally, 10, 29, and 23 genes were assigned to euAP2, euANT, and basalANT groups, respectively.

\section{Motif Composition and Gene Structure Analysis of TaAP2 Genes}

MEME was used to analyze protein motifs in the 62 AP2 protein sequences in wheat (Figure 2A). In total, 16 conserved motifs were identified and designated motif 1-16. Motifs 1, 2, 3 and 5 were found in almost all the members of the AP2 family.

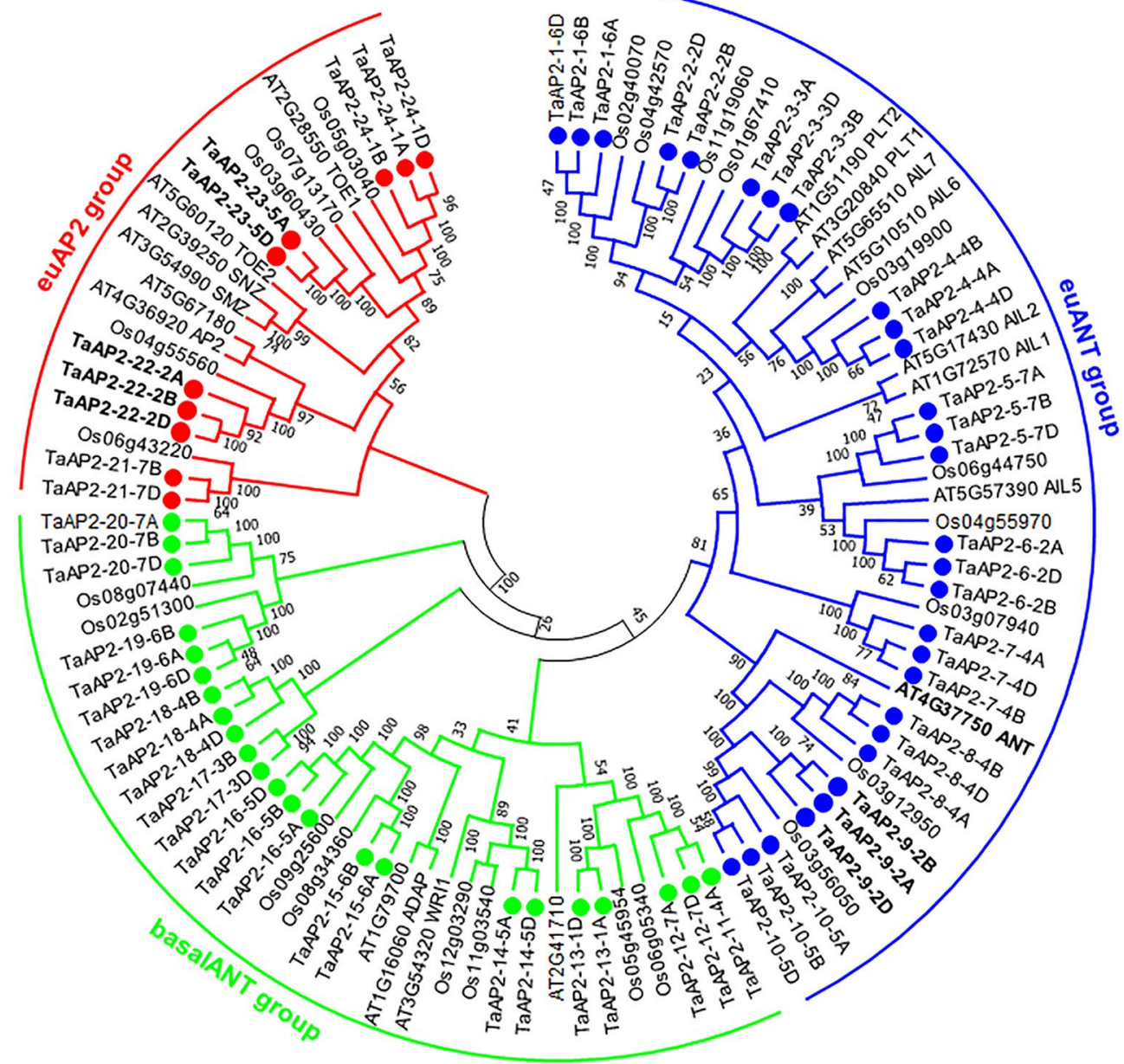

FIGURE 1 | Phylogenetic tree of AP2 proteins from wheat, Arabidopsis, and rice. The unrooted neighbor-joining (NJ) tree was constructed based on the AP2 proteins from wheat (62), Arabidopsis (18), and rice (23) using MEGA6.0. The numbers at nodes are bootstrap values after 1000 repetitions. The wheat AP2 TF proteins are named according to their positions in the tree. Each TaAP2 protein is indicated by a dot. The wheat AP2 proteins were classified into three groups, group euAP2, euANT, and basalANT, and they are represented by red, blue, and green, respectively. The three characterized TaAP2 genes and Arabidopsis ANT are highlighted in bold (TaAP2-23: Q; TaAP2-22: TaAP2; and TaPARG: TaAP2-9). 
$A$

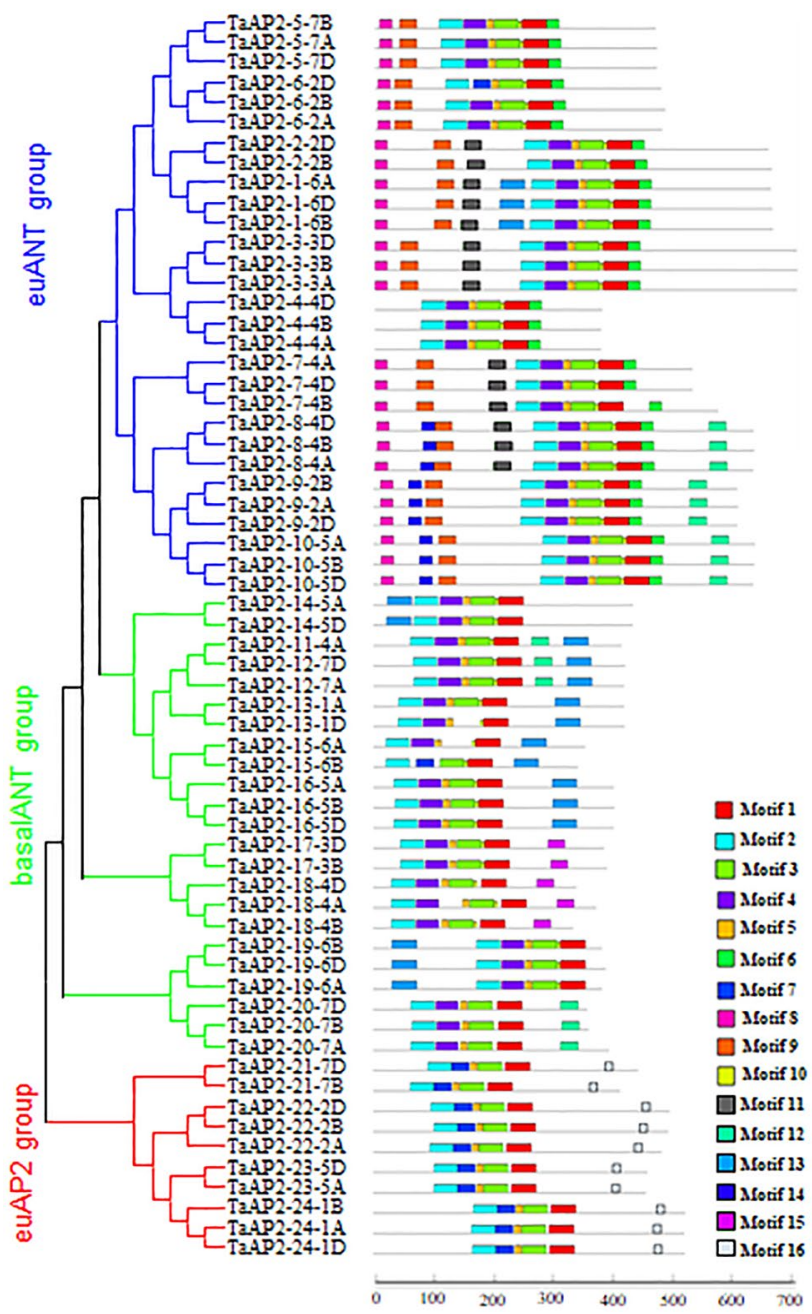

B

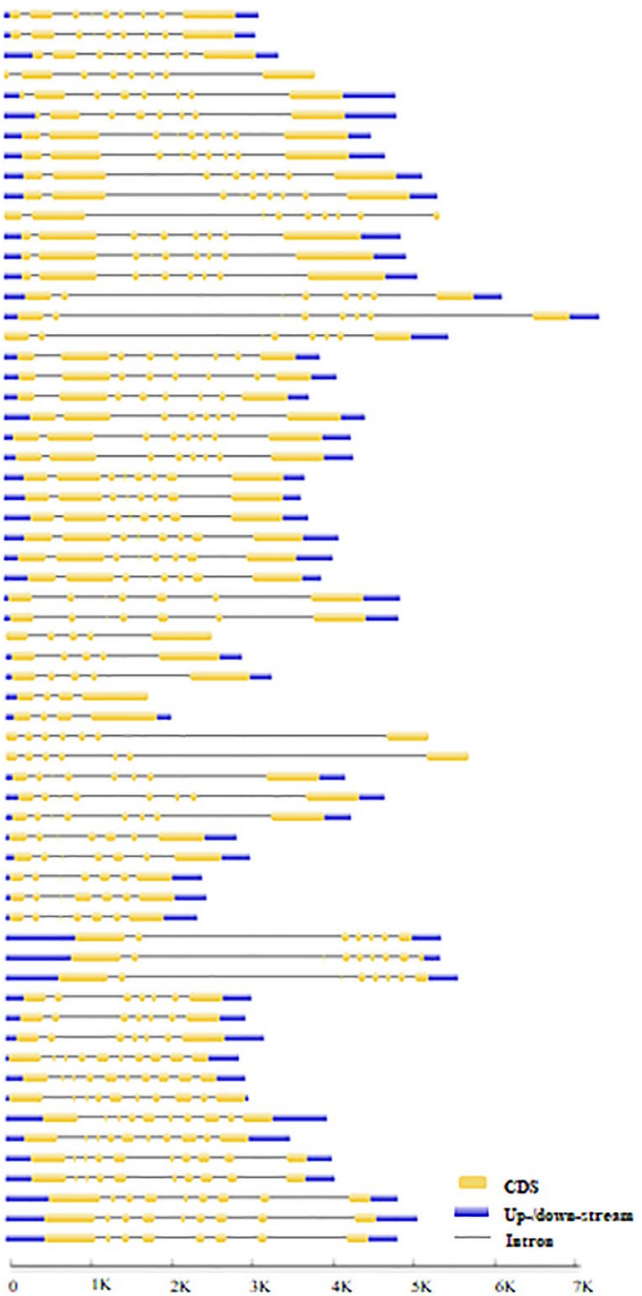

FIGURE 2 | Motif composition and exon-intron structures of TaAP2s. (A) Conserved motifs organization of TaAP2 proteins. Color blocks represent the position of motifs on corresponding proteins. Block sizes indicate the lengths of motifs. Gray lines represent non-conserved sequences. The relative position of each motif can be determined using the scale below. (B) Exon-intron structures of TaAP2 genes. Exons are represented by yellow boxes and introns were denoted by blank lines. The lengths of exons and introns could be estimated using the scale below.

Among them, Motifs 1 and 3 were parts of one AP2 domain, while motifs 2 and 5 were components of the other AP2 domain. The three groups of AP2 family proteins had their unique motif organizations. The euAP2 group had two motifs unique to its group, which were motifs 7 and 16. In groups euANT and basalANT, motif 7 was replaced by motif 4 , both of which were located on one AP2 domain. Motif 16 contained the predicted amino acid sequences (AAASSGF[S/P]) of the miR172 binding site. The majority of proteins in the basalANT group had the motif 13 and the remaining members in this group had motif 15 , and both of them were distinctive to the group. Motifs 6, 8 and 9 were exclusively contained in the proteins of the euANT group. The consensus sequences of these motifs are given in Figure S3 and Table S3.

In order to obtain more insights about gene evolution, the exon-intron organization of TaAP2 genes was investigated by aligning predicted coding sequences (CDS) against corresponding genomic sequences using the online service GSDS. As shown in Figure 2B and Table S2, the number of introns in the TaAP2 family genes ranged from 3 to 9 . Overall, highly similar gene structures were observed for the three groups of AP2 family genes, especially for euANT and euAP2 groups. The number of introns in the genes of the euANT and euAP2 groups were 7 or 8 , and 8 or 9 , respectively. Comparatively, the basalANT group exhibited diverse gene structures, with the number of introns ranging from 3 to 7 . Noticeably, all homologous genes in the AP2 family had the same number of introns.

\section{Genomic Distribution and Gene Duplication of TaAP2 Genes}

The TaAP2 genes were distributed unevenly among the 21 chromosomes of the wheat genome (Figure 3). The number of 


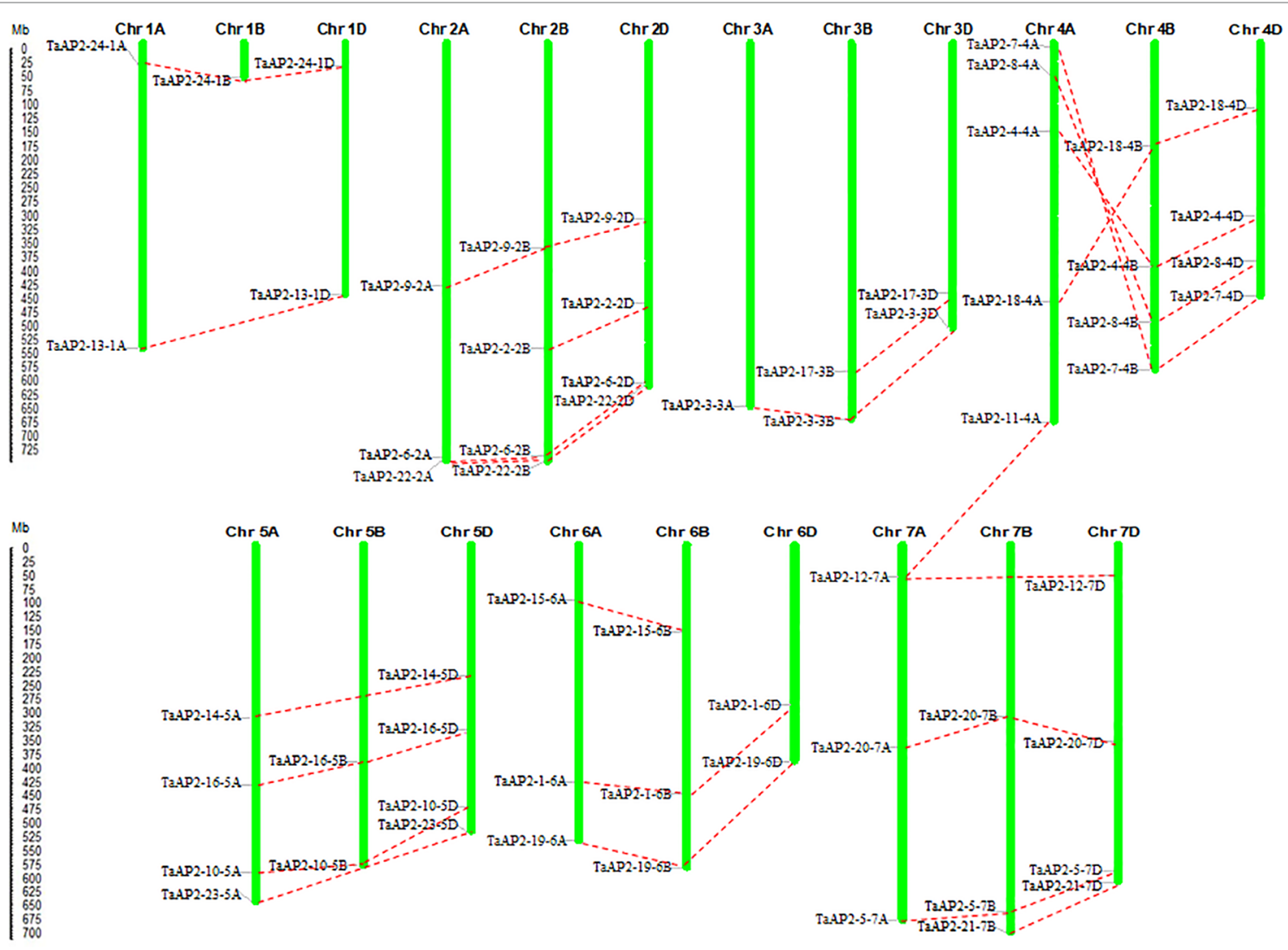

FIGURE 3 | Locations and duplication of the TaAP2 family genes on wheat chromosomes. The chromosomal position of each TaAP2 was mapped according to the physical positions of wheat genomes (Table S2). The chromosome number is labeled at the top of each chromosome. The scale is in mega bases (Mb). Putative TaAP2 homologous genes are depicted through dotted lines.

genes on each chromosome ranged from 1 (chromosomes $1 \mathrm{~B}$ and $3 \mathrm{~A}$ ) to 5 (chromosome $4 \mathrm{~A}$ ), with up to ten TaAP2 genes located on each of chromosome groups 2, 4, 5, and 7. Among the 62 TaAP2 genes, there were 21,19 , and 22 members distributed on wheat sub-genomes A, B, and D, respectively (Figure 3). In terms of gene duplication, there were 15 TaAP2 members (TaAP2$1,3,4,5,6,7,8,9,10,16,18,19,20,22$, and 24) containing three copies and eight members (TaAP2-2, 12, 13, 14, 15, 17, 21, and 23) containing two copies, and TaAP2-11 had only one copy which was on chromosomes 4A (Figure 3 and Table S4). Interestingly, TaAP2-11-4A had the highest sequence similarities to TaAP2-12-7A (95.3\%) and TaAP2-12-7D (96.3\%). Except for TaAP2-11-4A, no tandem or segmental duplication involving TaAP2s was discovered in the wheat genome. In addition, we found that TaAP2 genes on chromosome $4 \mathrm{~A}$ were reversed with their homologous genes on chromosomes $4 \mathrm{~B}$ and 4D (Figure 3).

\section{Expression Profiles of TaAP2 Genes in Various Wheat Tissues}

Gene expression pattern often has a strong correlation with gene functions. We investigated the expression levels of TaAP2s in various wheat tissues (leaf, root, spike, stem, and grain) across different developmental stages using the expression data in the WheatExp database. The expression data on $49 \mathrm{TaAP} 2$ genes were found in the database, while the information on the remaining 13 genes were missing (Figure 4). Of the $49 \mathrm{TaAP} 2 \mathrm{~s}, 11 \mathrm{TaAP} 2 \mathrm{~s}$ (TaAP2-7-4D, TaAP2-10-5D, TaAP2-19-6A/B/D, TaAP2-20$7 A / B / D, T a A P 2-22-2 A / B$, and $T a A P 2-23-5 A$ ) were expressed in all tissues at different developmental stages, whereas $6 \mathrm{TaAP} 2 \mathrm{~s}$ (TaAP2-4-4B/D, TaAP2-9-2A, TaAP2-11-4A, TaAP2-12-7A, and TaAP2-13-1D) had almost no expression in any tested tissues (Figure 4). The remaining $32 \mathrm{TaAP} 2 \mathrm{~s}$ were expressed in one or several specific tissue(s). The expression patterns of TaAP2 genes had similarities and differences within a group. The majority of genes in the euAP2 and basalANT groups had relatively high levels of expression in all or most tissues examined, whereas most genes in the euANT group were mainly expressed in roots and spikes (Figure 4). In terms of the gene expression patterns among homologous genes, most homologous genes shared a similar expression pattern, including TaAP2-5, -6, -2, -1, -3, -4, -8, -9, $-14,-19,-20,-22,-23$, and -24 . In contrast, different expression profiles were observed for TaAP2-7 and TaAP2-10.

To validate the gene expression profiles obtained from the WheatExp database, wheat roots, stems, leaves, and spikes were collected for RNA extraction and qPCR analysis. According to TaAP2-5 gene sequences, we designed sub-genome-specific primer sets for TaAP2-5A, TaAP2-5B and TaAP2-5D. The specificity of each primer set was validated in Chinese Spring 


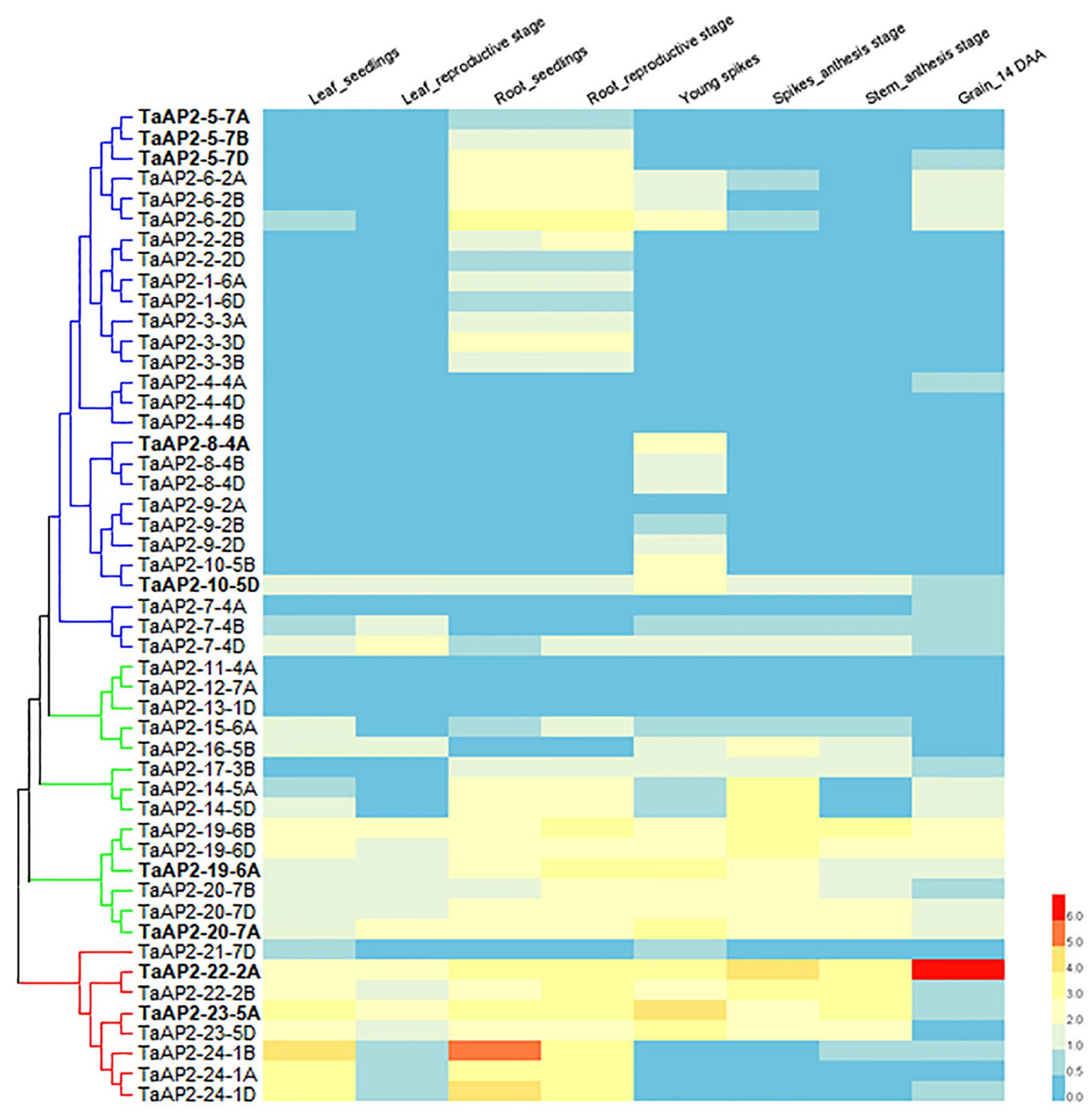

FIGURE 4 | Expression patterns of 49 TaAP2 genes in various tissues at different developmental stages in wheat. The tissues and developmental stages examined are indicated at the top of the heat map. $\log _{2}$ transformed (FPKM +1$)$ expression values were used to create the heat map. The highest level of expression is represented by red $(100 \%)$, while the low level is represented by blue $(0 \%)$.

nullisomic/tetrasomic (NT) lines (Figure 5A). As shown in Figure 5B, TaAP2-5s was dominantly expressed in roots, and the expression level of TaAP2-5D was higher than those of TaAP2-5B and TaAP2-5A. The expression patterns of TaAP2-5s shown here resemble those in Figure 4. The consistent results between qPCR and public data were also observed for TaAP2-8-4A, TaAP210-5D, TaAP2-19-6A, TaAP2-20-7A, TaAP2-22-2A, and TaAP223-5A (Figures 5C-H and Figure 4).

\section{Overexpression of TaAP2-10-5D Led to Enlarged Plant Sizes in Arabidopsis}

TaAP2-10-5D was phylogenetically close to Arabidopsis ANT (AT4G37750) (Figure 1). Moreover, TaAP2-10-5D was ubiquitously expressed in different tissues, which was the same as Arabidopsis ANT. Thus, we hypothesized that TaAP2-10-5D might play an important role in growth and development of wheat. To uncover the potential function of TaAP2-10-5D, we produced Arabidopsis plants overexpressing TaAP2-10-5D under the control of the MAS promoter (Figure 6A). The expression levels of TaAP2-10-5D were detected in $6 \mathrm{~T}_{3}$ homozygous transgenic Arabidopsis lines by qPCR, and three independent lines with the highest expression levels (L1, L2, and L3) were selected for further analyses (Figure 6B).

When grown for 7 days, the TaAP2-10-5D transgenic seedlings exhibited longer roots than WT plants (Figures 6C, D). The seedling size of the transgenic plants at $28 \mathrm{~d}$ was significantly larger than that of the WT (Figure 6C, E). Additionally, TaAP210-5D overexpression shortened the flowering time and enlarged the flower dimension in transgenic Arabidopsis plants (Figures 7A, B). Furthermore, plant height, fresh weight, silique length, 
A

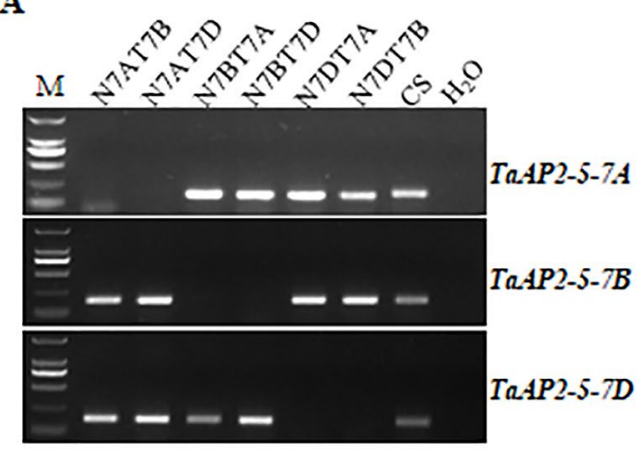

B

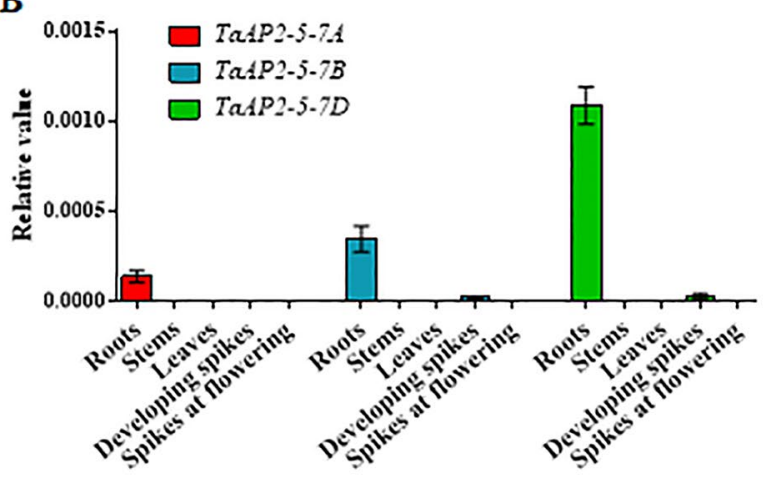

C
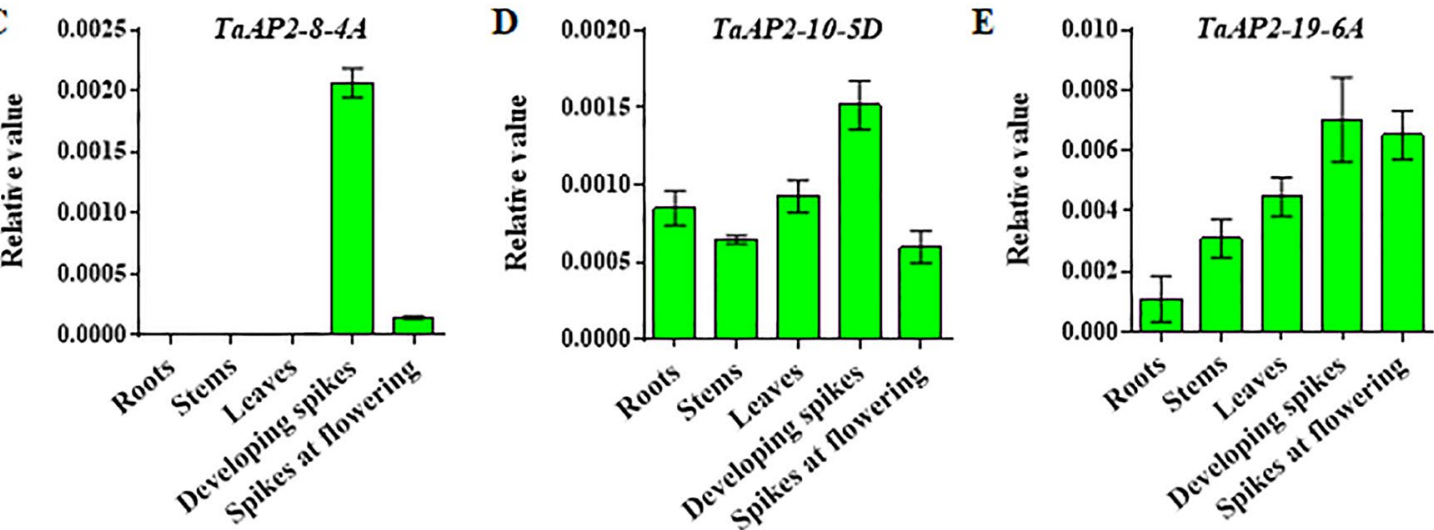

F

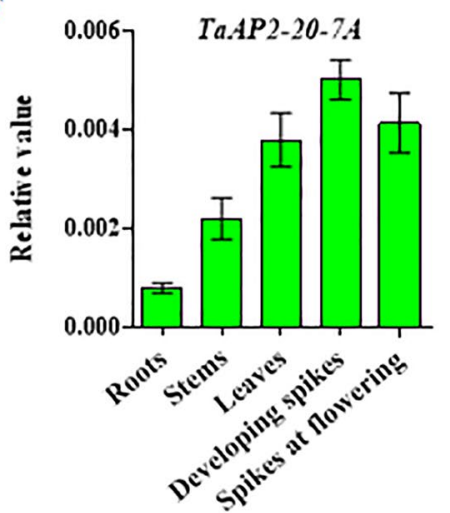

G

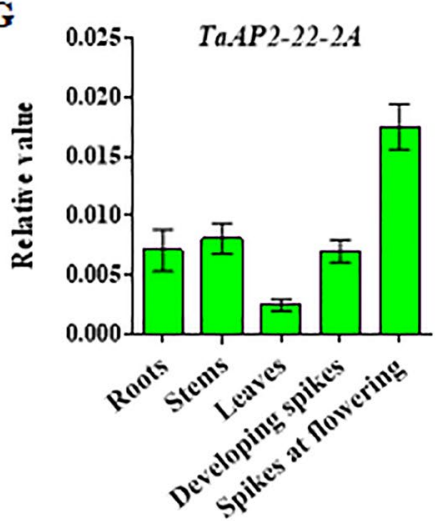

H

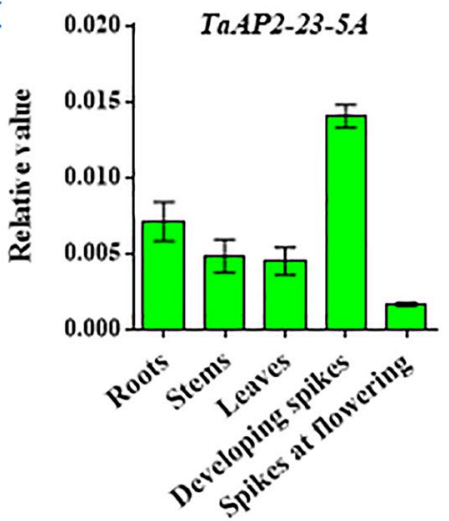

FIGURE 5 | QPCR verification of the expression levels of several TaAP2 genes in different wheat tissues, including roots, stems, leaves, developing spikes, and spikes at flowering. (A) Verification of the sub-genome specificity of primer pairs for TaAP2-5 genes in Chinese Spring nullisomic/tetrasomic (NT) lines. (B) Expression levels of TaAP2-5A, TaAP2-5B, and TaAP2-5D revealed by qPCR. (C-H) Expression levels of TaAP2-8-4A (C), TaAP2-10-5D (D), TaAP2-19-6A (E), TaAP2-20-7A (F), TaAP2-22-2A (G), and TaAP2-23-5A (H) revealed by qPCR. Data were normalized by the wheat $\beta$-actin gene using the $2-\triangle \mathrm{Ct}$ method. Bars indicate standard deviations of three biological replicates.

and silique number of the transgenic TaAP2-10-5D plants were markedly increased compared with those of the WT plants at the mature stage (Figures 7C-F). For seed number per silique, no significant differences were observed between transgenic and WT plants (Figure 7G). To explore the potential factors leading to the bigger plant size of transgenic plants, we examined the leaf epidermal cell number per unit area and the leaf epidermal cell size on the 5th leaves of the 28-day-old WT and transgenic plants. No striking differences were found for both cell number per unit area and cell size (Figures 8A, B), suggesting that the enlarged plant size in the transgenic plants was resulted from cell proliferation.

\section{DISCUSSION}

Genome-wide analysis of a gene family represents an effective approach to the characterization of plant gene functions, and it facilitates the study of the evolution of genes and genomes 

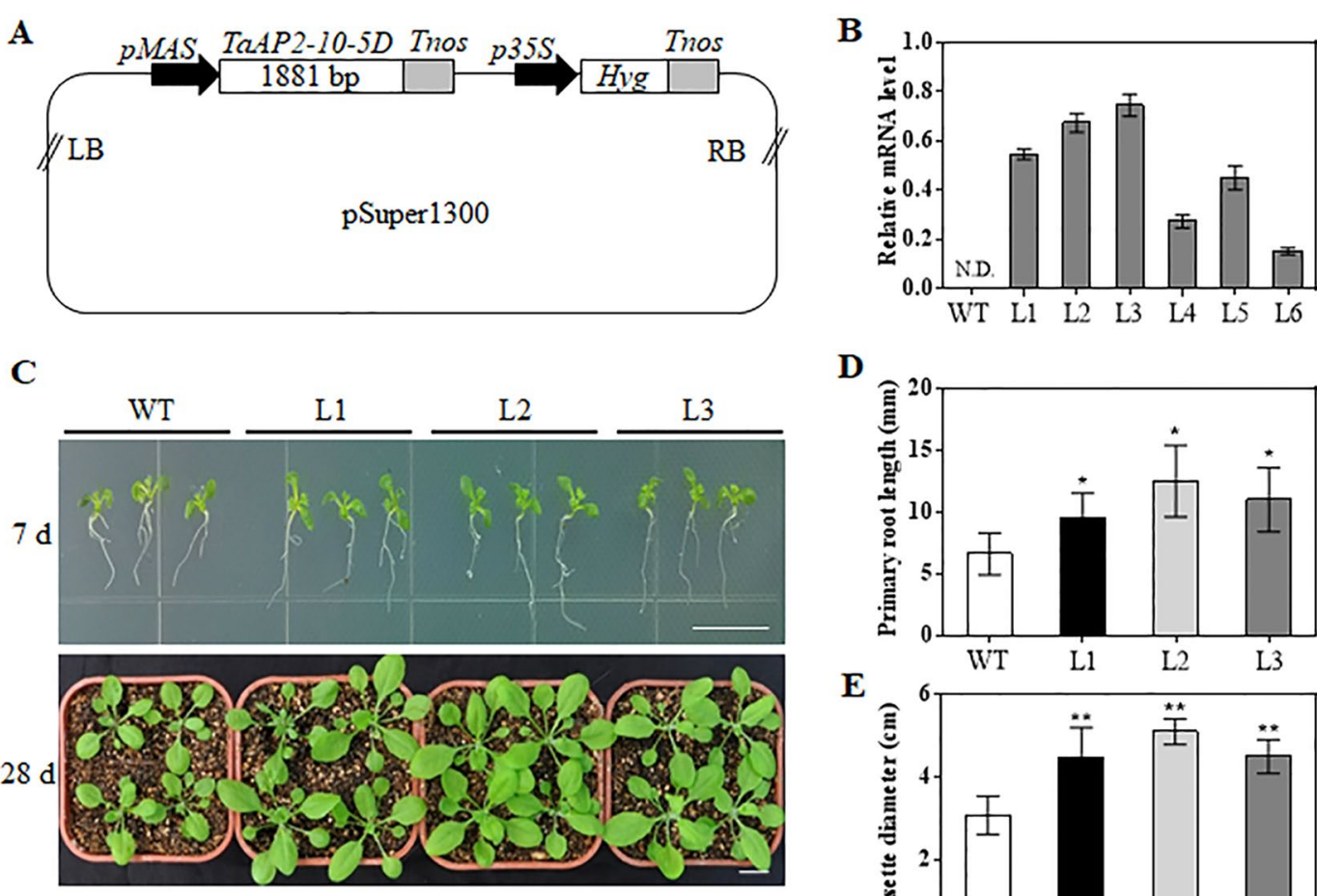

D

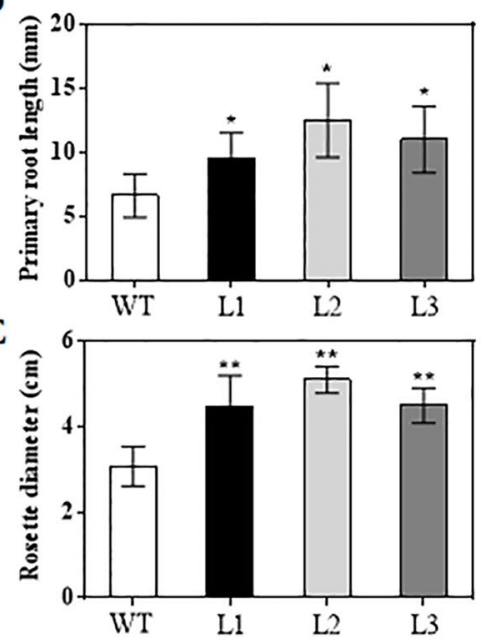

FIGURE 6 | Morphological differences between WT and TaAP2-10-5D transgenic plants at seedling stages. (A) Schematic diagram of the construct containing the TaAP2-10-5D expression cassette. LB, left border; pMAS, mannopine synthase promoter; Thos, nopaline synthase gene (NOS) terminator; p35S, cauliflower mosaic virus $35 S$ promoter; Hyg, Hygromycin-resistance gene; RB, right border. (B) Transcript levels of TaAP2-10-5D in $\mathrm{T}_{3}$ transgenic Arabidopsis lines revealed by qPCR analysis. Bars indicate standard deviations of three biological replicates. N.D., Not Detected. (C) Phenotype of WT and TaAP2-10-5D transgenic seedlings grown for 7 and 28 days. (D) Root lengths of the WT and TaAP2-10-5D transgenic plants at the 7 day after sowing. (E) Rosette diameters of 28-day-old WT and TaAP2-10-5D transgenic plants. Bars $=10 \mathrm{~mm}$. Mean values were calculated from 10-20 biological replicates, with error bars representing standard deviations. Statistically significant differences are indicated: ${ }^{*}, P<0.05 ;{ }^{* \star}, P<0.01$ (Student's $t$-test).

(Zhang et al., 2015a). In the present study, 62 AP2 genes were identified from a genome-wide search against the current version of the wheat genome data. Phylogenetic analyses revealed that all the three phylogenetic groups (euANT, basalANT, and euAP2) contained more members in wheat than in Arabidopsis or rice. For instance, in the basalANT group, wheat had nearly 10 times as many AP2 genes as their Arabidopsis counterparts (Figure 1 and Figure S1), which may be the result of its allohexaploidy and a complex evolutionary process.

Domains and motifs of TFs were proven to be involved in various activities including protein interaction, transcriptional activity, and DNA binding (Liu et al., 1999). Motif analysis revealed 16 conserved motifs in TaAP2 proteins, and motifs 1 , $2,3,4,5$, and 7 were included in AP2 domains. Among them, motifs $1,2,3$, and 5 were conserved in all the TaAP2 proteins identified in this study, while motifs 4 and 7 were specific to euANT/basalANT and euAP2 groups, respectively. The motif 4 contains a 10-aa insertion while motif 7 lacks a 10-aa insertion. The10-aa insertion has been reported to be a major difference between euANT/basalANT and euAP2 groups (Kim et al., 2006; Horstman et al., 2014). Another difference is that all the euAP2 proteins contained the unique motif 16 , which covered the predicted amino acid sequences of miR172 binding sites (Figure 2A and Figure S4). The miR172 is known to regulate the euAP2 TFs through transcript cleavage in Arabidopsis, rice, and Brassica napus (Chen, 2004; Tang et al., 2007; Wang et al., 2019b), suggesting that miR172 binding sites are conserved in euAP2 genes in plants. Further, we demonstrated that motifs 6 , 8 , and 9 were exclusively contained in the proteins of the euANT group. Noticeably, motifs 4,8 and 9 cover motifs euANT1 (NSC[K/R][K/R]EGQ[T/S]R), euANT2 (WLGFSLS), and euANT3 (PKLEDFLG), respectively, which were reported to be characteristic of the euANT group (Figure S2, Kim et al., 2006). In addition, we found that TaAP2-8, TaAP2-9, and TaAP2-10, which were putative homologs of AtANT, contained a unique motif (motif 14) only present in their sequences (Figure 2A and Figure S5). Investigation into this motif might reveal new biological functions and regulation mechanisms of ANT genes. It has been suggested that TFs that share unique motifs in a cluster are likely to possess similar functions. For example, in the ERF proteins family, the ERF-associated amphiphilic repression (EAR) motif ((L/F)DLN(L/F)XP) was specifically present in the VIII group genes, and it was essentially required for a repression function of ERF proteins (Nakano et al., 2006). In this study, we 
A
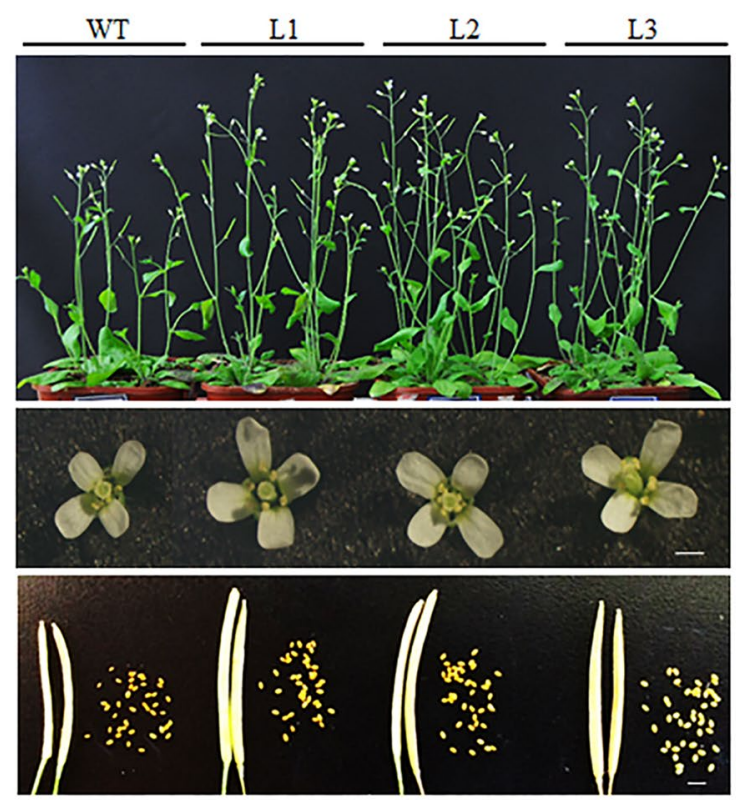

E

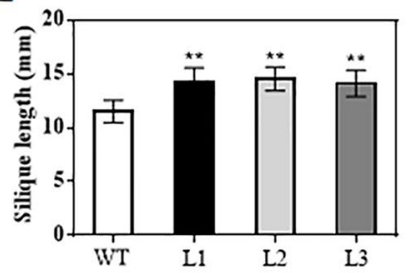

F

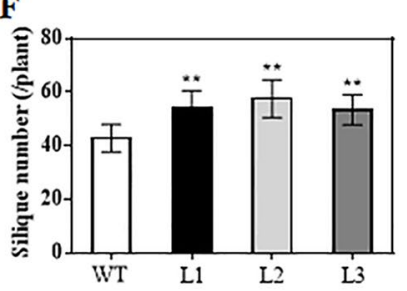

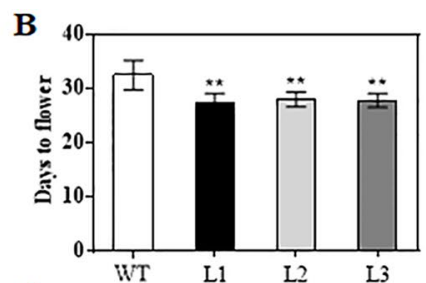

C
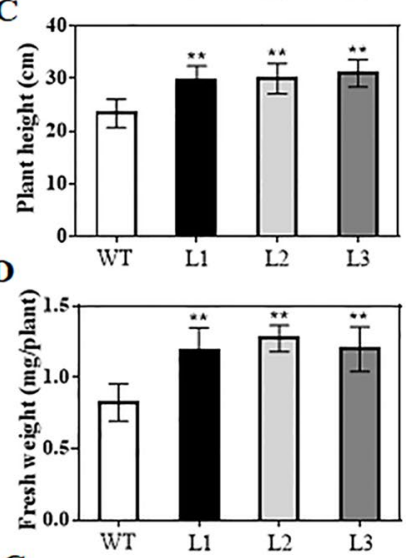

G

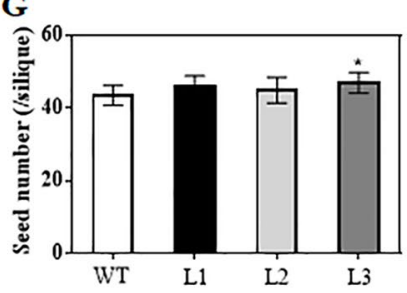

FIGURE 7 | Phenotypic analyses of the TaAP2-10-5D transgenic Arabidopsis at reproductive stages. (A) Comparisons of the whole plants, flower sizes and silique sizes between WT and transgenic plants. Bar = $1 \mathrm{~mm}$. (B-G) Flowering times (B), plant height (C), fresh weight (D), silique length (E), silique number (F) and seed number per silique (G) of WT and transgenic plants. Mean values were calculated from 10-20 biological replicates, with error bars representing standard deviations. Statistically significant differences are indicated: * $P<0.05 ;{ }^{\star *}, P<0.01$ (Student's $t$-test).

found that TaAP2-23/24 also had the EAR motif (Figure S4). Liu et al. (2018) demonstrated that the EAR motif of Q protein, which is TaAP2-23, is essential for the transcriptional repression activity of Q protein.

Gene structure analysis could provide important information about the gene function and evolution. We found that most TaAP2 genes shared a similar exon/intron structure within the same phylogenetic group although some differences were also observed (Figure 2B). The genes in the basalANT group have more diversified structures than those in euANT and euAP2 groups due to their various numbers of introns. In detail, the basalANT genes had 3 to 7 introns, while in the euANT and euAP2 groups, the genes had 7 to 8 and 8 to 9 introns, respectively. It has been revealed that intron gain or loss is the result of selection pressures during evolution in plants, and genes tend to evolve into diverse exon-intron structures and perform distinct functions (Mattick, 1994; Wang et al., 2016b). Our results imply that gene differentiation might have occurred in the wheat TaAP2 family to accomplish different biological functions under selection pressure during the wheat genome formation and evolution.
Gene duplication is frequently observed in plant genomes, arising from polyploidization or through tandem and segmental duplication (Zhang, 2003). The wheat genome (BBAADD) has undergone two rounds of genome duplication early in its evolution, which led to a complex genome consisting of three related sub-genomes that were derived from three different diploid species (Feldman and Levy, 2012). Among the 24 homologous members of TaAP2 genes identified in this study, a total of 21,19 , and 22 TaAP2 genes were found on the A, B, and D sub-genomes, respectively (Figure 3 and Table S4). This result implied that gene loss might have occurred in the wheat AP2 gene family, resulting in the loss of some homologous copies. The retention and distribution of TaAP2s on homologous chromosomes provide invaluable information for better understanding the interaction and polyploidization of wheat chromosomes. Furthermore, it is worth noting that there was the least number of TaAP2 genes on the subgenome $\mathrm{B}$. This observation strengthens the notion that initial gene loss may have occurred in the B sub-genome following tetraploidy to reduce functional redundancy, and the loss maintained following the formation of the hexaploid 
A

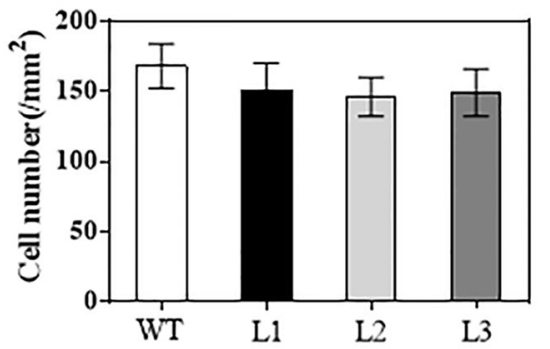

B

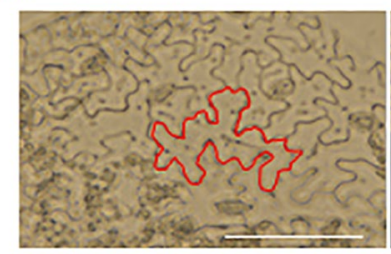

WT

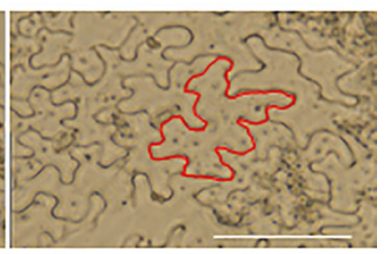

L1
FIGURE 8 | Analysis of cell number (A) and cell size (B) in TaAP2-10-5D transgenic plants. Mean values were calculated from five biological replicates. Bars $=50 \mu \mathrm{m}$. Error bars represent standard deviations.

wheat $\sim 8000-10,000$ years ago (Feldman, 2001; Wang et al., 2016b). One such example is TaAP2-23, which is the well-known $Q$ gene, the homologous copy of TaAP2-23 on the B sub-genome was missing (Table S2). Previous studies revealed that the $q$ allele on wheat homologous chromosome 5B became a pseudogene after allotetraploidization (Zhang et al., 2011). Analysis of gene duplication found that TaAP211-4A had high sequence similarities with TaAP2-12-7A and TaAP2-12-7D. This finding was in accordance with previous reports that non-homologous translocation between chromosomes $4 \mathrm{~A}$ and $7 \mathrm{~B}$ occurred in hexaploid wheat (Devos et al., 1995; Berkman et al., 2012). Therefore, TaAP2-11-4A is probably to be the nonexistent TaAP2-12-7B. Except for TaAP2-11-4A, no tandem or segmental duplication involving $T a A P 2 s$ was discovered in the wheat genome, suggesting that the expansion of the wheat AP2 gene family was originated from polyploidization. In addition, we found that the TaAP2 genes on chromosome 4A were reversed with their homologous genes on chromosomes $4 \mathrm{~B}$ and $4 \mathrm{D}$ (Figure 3). This result reinforce the previous findings that the modern chromosome $4 \mathrm{~A}$ arm has been reversed in the hexaploid wheat (Ma et al., 2015).

The expression pattern of a gene is closely correlated with its function. Understanding the tendency of gene expression in different gene clusters will help narrow down candidate genes. To understand the potential functions of AP2 family genes in wheat, we analyzed the expression of these genes in various wheat tissues. As shown in Figure 4, the members in the same group had similar expression patterns. For instance, TaAP2-1, TaAP2-2, and TaAP2-3, all belonging to the euANT group, were almost exclusively expressed in the roots, suggesting a potential role in the development of roots. On the other hand, however, there is possibility that these three genes are functionally redundant. A previous report demonstrated that Arabidopsis AIL5, AIL6, and AIL7 genes were all expressed in developing flowers, and that they had partly overlapping functions with the AtANT gene in Arabidopsis flower development (Krizek, 2015). In addition, we found that most homologous genes shared a similar expression pattern during development. However, different expression profiles were observed for TaAP2-7 and TaAP2-10, suggesting that these genes might have undergone subfunctionalization or neofunctionalization in the wheat evolutionary process.

Accumulating evidence showed that AP2 family genes play pivotal roles in regulating plant growth and development (Scheres and Krizek, 2018; Dipp-Alvarez and Cruz-Ramirez, 2019; Jiang et al., 2019; Wang et al., 2019a). Arabidopsis AtANT was one of the first genes in the family that were functionally characterized; it regulates organ size, floral meristem patterning, and plant defense pathways (Elliott et al., 1996; Krizek et al., 2016). In wheat, three TaAP2 family members, TaAP2-8, TaAP2-9, and TaAP2-10, were grouped into the same cluster with AtANT (Figure 1). Among them, TaAP2-10-5D had a similar expression pattern with AtANT (Figures 4 and 5D), thus, TaAP2-10-5D was selected for further functional characterization. Overexpression of TaAP2-10-5D in Arabidopsis enlarged organ sizes via increased cell numbers rather than cell sizes (Figure 8), implying that TaAP2-10-5D positively regulates cell proliferation. This result was consistent with the function of the AtANT gene in Arabidopsis. It will be interesting to reveal a more detailed function of TaAP2-10-5D in wheat as well as the underlying molecular basis, via wheat transgenesis and CRISPR/Cas genome editing in combination with modern phenotypic analyses and next-generation sequencing (NGS).

\section{DATA AVAILABILITY STATEMENT}

All datasets generated for this study are included in the manuscript/Supplementary Files.

\section{AUTHOR CONTRIBUTIONS}

YZ carried out the experiments, analyzed the data, and drafted the manuscript. HB, ZX, and HP provided the idea and instructed the research work. RM and DX provided assistance to perform experiments and collect data. HB revised the manuscript. All authors have read and approved the final manuscript.

\section{FUNDING}

This work was supported by the National Natural Science Foundation of China (31801363).

\section{SUPPLEMENTARY MATERIAL}

The Supplementary Material for this article can be found online at: https://www.frontiersin.org/articles/10.3389/fpls.2019.01286/ full\#supplementary-material 


\section{REFERENCES}

Aida, M., Beis, D., Heidstra, R., Willemsen, V., Blilou, I., Galinha, C., et al. (2004). The PLETHORA genes mediate patterning of the Arabidopsis root stem cell niche. Cell 119, 109-120. doi: 10.1016/j.cell.2004.09.018

Appels, R., Eversole, K., Feuillet, C., Keller, B., Rogers, J., Stein, N., et al. (2018). Shifting the limits in wheat research and breeding using a fully annotated reference genome. Science 361, eaar7191. doi: 10.1126/science.aar7191

Aukerman, M. J., and Sakai, H. (2003). Regulation of flowering time and floral organ identity by a MicroRNA and its APETALA2-like target genes. Plant Cell 15, 2730-2741. doi: 10.1105/tpc.016238

Aya, K., Hobo, T., Sato-Izawa, K., Ueguchi-Tanaka, M., Kitano, H., and Matsuoka, M. (2014). A novel AP2-type transcription factor, SMALL ORGAN SIZE1, controls organ size downstream of an auxin signaling pathway. Plant Cell Physiol. 55, 897-912. doi: 10.1093/pcp/pcu023

Berkman, P. J., Skarshewski, A., Manoli, S., Lorenc, M. T., Stiller, J., Smits, L., et al. (2012). Sequencing wheat chromosome arm 7BS delimits the 7BS/4AL translocation and reveals homoeologous gene conservation. Theor. Appl. Genet. 124, 423-432. doi: 10.1007/s00122-011-1717-2

Boutilier, K., Offringa, R., Sharma, V. K., Kieft, H., Ouellet, T., Zhang, L., et al. (2002). Ectopic expression of BABY BOOM triggers a conversion from vegetative to embryonic growth. Plant Cell 14, 1737-1749. doi: 10.1105/tpc.001941

Bowman, J. L., Smyth, D. R., and Meyerowitz, E. M. (1989). Genes directing flower development in Arabidopsis. Plant Cell 1, 37-52. doi: 10.1105/tpc.1.1.37

Chen, X. (2004). A microRNA as a translational repressor of APETALA2 in Arabidopsis flower development. Science 303, 2022-2025. doi: 10.1126/science. 1088060

Chuck, G., Meeley, R., and Hake, S. (2008). Floral meristem initiation and meristem cell fate are regulated by the maize AP2 genes ids1 and sid1. Development 135 , 3013-3019. doi: 10.1242/dev.024273

Clough, S. J., and Bent, A. F. (1998). Floral dip: a simplified method for Agrobacterium-mediated transformation of Arabidopsis thaliana. Plant J. 16, 735-743. doi: 10.1046/j.1365-313x.1998.00343.x

Coen, E. S., and Meyerowitz, E. M. (1991). The war of the whorls-genetic interactions controlling flower development. Nature 353, 31-37. doi: 10.1038/353031a0

Deng, W., Wang, Y., Liu, Z., Cheng, H., and Xue, Y. (2014). HemI: a toolkit for illustrating heatmaps. PLoS One 9, e111988. doi: 10.1371/journal.pone.0111988

Devos, K. M., Dubcovsky, J., Dvorák, J., Chinoy, C. N., and Gale, M. D. (1995). Structural evolution of wheat chromosomes $4 \mathrm{~A}, 5 \mathrm{~A}$, and 7B and its impact on recombination. Theor. Appl. Genet. 91, 282-288. doi: 10.1007/BF00220890

Dipp-Alvarez, M., and Cruz-Ramirez, A. (2019). A phylogenetic study of the ANT Family points to a preANT gene as the ancestor of basal and euANT transcription factors in land plants. Front. Plant Sci. 10, 17. doi: 10.3389/ fpls.2019.00017

Drews, G. N., Bowman, J. L., and Meyerowitz, E. M. (1991). Negative regulation of the Arabidopsis homeotic gene agamous by the APETALA2 product. Cell 65, 991-1002. doi: 10.1016/0092-8674(91)90551-9

Elliott, R. C., Betzner, A. S., Huttner, E., Oakes, M. P., Tucker, W. Q., Gerentes, D., et al. (1996). AINTEGUMENTA, an APETALA2-like gene of Arabidopsis with pleiotropic roles in ovule development and floral organ growth. Plant Cell 8, 155-168. doi: 10.1105/tpc.8.2.155

Feldman, M. (2001). Origin of cultivated wheat. Genome 8, 220-232. doi: 10.1139/ g66-028

Feldman, M., and Levy, A. A. (2012). Genome evolution due to allopolyploidization in wheat. Genetics 192, 763-774. doi: 10.1534/genetics.112.146316

Gil-Humanes, J., Piston, F., Martin, A., and Barro, F. (2009). Comparative genomic analysis and expression of the APETALA2-like genes from barley, wheat, and barley-wheat amphiploids. BMC Plant Biol. 9, 66. doi: 10.1186/1471-2229-9-66

Greenwood, J. R., Finnegan, E. J., Watanabe, N., Trevaskis, B., and Swain, S. M. (2017). New alleles of the wheat domestication gene $Q$ reveal multiple roles in growth and reproductive development. Development 144, 1959-1965. doi: 10.1242/dev.146407

Horstman, A., Willemsen, V., Boutilier, K., and Heidstra, R. (2014). AINTEGUMENTALIKE proteins: hubs in a plethora of networks. Trends Plant Sci. 19, 146-157. doi: 10.1016/j.tplants.2013.10.010

Hu, B., Jin, J., Guo, A. Y., Zhang, H., Luo, J., and Gao, G. (2015). GSDS 2.0: an upgraded gene feature visualization server. Bioinformatics 31, 1296-1297. doi: 10.1093/bioinformatics/btu817
Jiang, L., Ma, X., Zhao, S., Tang, Y., Liu, F., Gu, P., et al. (2019). The APETALA2Like transcription factor SUPERNUMERARY BRACT controls rice seed shattering and seed size. Plant Cell 31, 17-36. doi: 10.1105/tpc.18.00304

Jofuku, K. D., Omidyar, P. K., Gee, Z., and Okamuro, J. K. (2005). Control of seed mass and seed yield by the floral homeotic gene APETALA2. Proc. Natl. Acad. Sci. U. S. A. 102, 3117-3122. doi: 10.1073/pnas.0409893102

Kawahara, Y., de la Bastide, M., Hamilton, J. P., Kanamori, H., McCombie, W. R., Ouyang, S., et al. (2013). Improvement of the Oryza sativa Nipponbare reference genome using next generation sequence and optical map data. Rice (N Y) 6, 4. doi: 10.1186/1939-8433-6-4

Kim, S., Soltis, P. S., Wall, K., and Soltis, D. E. (2006). Phylogeny and domain evolution in the APETALA2-like gene family. Mol. Biol. Evol. 23, 107-120. doi: $10.1093 / \mathrm{molbev} / \mathrm{msj} 014$

Klucher, K. M., Chow, H., Reiser, L., and Fischer, R. L. (1996). The AINTEGUMENTA gene of Arabidopsis required for ovule and female gametophyte development is related to the floral homeotic gene APETALA2. Plant Cell 8, 137-153. doi: 10.1105/tpc.8.2.137

Krizek, B. (2009). AINTEGUMENTA and AINTEGUMENTA-LIKE6 act redundantly to regulate Arabidopsis floral growth and patterning. Plant Physiol. 150, 19161929. doi: $10.1104 /$ pp.109.141119

Krizek, B. A. (2015). AINTEGUMENTA-LIKE genes have partly overlapping functions with AINTEGUMENTA but make distinct contributions to Arabidopsis thaliana flower development. J. Exp. Bot. 66, 4537-4549. doi: 10.1093/jxb/erv224

Krizek, B. A., Bequette, C. J., Xu, K., Blakley, I. C., Fu, Z. Q., Stratmann, J. W., et al. (2016). RNA-Seq links the transcription factors AINTEGUMENTA and AINTEGUMENTA-LIKE6 to cell wall remodeling and plant defense pathways. Plant Physiol. 171, 2069-2084. doi: 10.1104/pp.15.01625

Kulkarni, M., Soolanayakanahally, R., Ogawa, S., Uga, Y., Selvaraj, M. G., and Kagale, S. (2017). Drought response in wheat: key genes and regulatory mechanisms controlling root system architecture and transpiration efficiency. Front Chem 5, 106. doi: 10.3389/fchem.2017.00106

Kunst, L., Klenz, J. E., Martinezzapater, J., and Haughn, G. W. (1989). AP2 gene determines the identity of perianth organs in flowers of Arabidopsis thaliana. Plant Cell 1, 1195-1208. doi: 10.2307/3868917

Larkin, M. A., Blackshields, G., Brown, N. P., Chenna, R., McGettigan, P. A., McWilliam, H., et al. (2007). Clustal W and Clustal X version 2.0. Bioinformatics 23, 2947-2948. doi: 10.1093/bioinformatics/btm404

Lee, D., and An, G. (2012). Two AP2 family genes, SUPERNUMERARY BRACT (SNB) and OSINDETERMINATE SPIKELET 1 (OSIDS1), synergistically control inflorescence architecture and floral meristem establishment in rice. Plant J. 69, 445-461. doi: 10.1111/j.1365-313X.2011.04804.x

Lee, Y. S., Lee, D. Y., Cho, L. H., and An, G. (2014). Rice miR172 induces flowering by suppressing OsIDS1 and SNB, two AP2 genes that negatively regulate expression of Ehd1 and florigens. Rice (N Y) 7, 31. doi: 10.1186/ s12284-014-0031-4

Letunic, I., Doerks, T., and Bork, P. (2015). SMART: recent updates, new developments and status in 2015. Nucleic Acids Res. 43, D257-D260. doi: 10.1093/nar/gku949

Li, B., Li, Q., Mao, X., Li, A., Wang, J., Chang, X., et al. (2016). Two novel AP2/ EREBP transcription factor genes TaPARG have pleiotropic functions on plant architecture and yield-related traits in common wheat. Front. Plant Sci. 7, 1191. doi: $10.3389 /$ fpls.2016.01191

Liu, L., White, M. J., and MacRae, T. H. (1999). Transcription factors and their genes in higher plants: functional domains, evolution and regulation. Eur. J. Biochem. 262, 247-257. doi: 10.1046/j.1432-1327.1999.00349.x

Liu, P., Liu, J., Dong, H., and Sun, J. (2018). Functional regulation of $Q$ by microRNA172 and transcriptional co-repressor TOPLESS in controlling bread wheat spikelet density. Plant Biotechnol. J. 16, 495-506. doi: 10.1111/pbi.12790

Livak, K. J., and Schmittgen, T. D. (2001). Analysis of relative gene expression data using real-time quantitative PCR and the 2(-Delta Delta C(T)) Method. Methods 25, 402-408. doi: 10.1006/meth.2001.1262

Ma, J., Gao, S., Stiller, J., Jiang, Q., Lan, X., Liu, Y., et al. (2015). Identification of genes bordering breakpoints of the pericentric inversions on $2 \mathrm{~B}, 4 \mathrm{~B}$, and $5 \mathrm{~A}$ in bread wheat (Triticum aestivum L.). Genome 58, 385-390. doi: 10.1139/ gen-2015-0060

Mandel, M. A., Bowman, J. L., Kempin, S. A., Ma, H., Meyerowitz, E. M., and Yanofsky, M. F. (1992). Manipulation of flower structure in transgenic tobacco. Cell 71, 133-143. doi: 10.1016/0092-8674(92)90272-E 
Marchler-Bauer, A., Bo, Y., Han, L., He, J., Lanczycki, C. J., Lu, S., et al. (2017). CDD/SPARCLE: functional classification of proteins via subfamily domain architectures. Nucleic Acids Res. 45, D200-D203. doi: 10.1093/nar/ gkw1129

Mathieu, J., Yant, L. J., Murdter, F., Kuttner, F., and Schmid, M. (2009). Repression of flowering by the miR172 target SMZ. PLoS Biol. 7, e1000148. doi: 10.1371/ journal.pbio.1000148

Mattick, J. S. (1994). Introns: evolution and function. Curr. Opin. Genet. Dev. 4, 823-831. doi: 10.1016/0959-437X(94)90066-3

Moose, S. P., and Sisco, P. H. (1996). Glossy15, an APETALA2-like gene from maize that regulates leaf epidermal cell identity. Gene. Dev. 10, 3018-3027. doi: 10.1101/gad.10.23.3018

Nakano, T., Suzuki, K., Fujimura, T., and Shinshi, H. (2006). Genome-wide analysis of the ERF gene family in Arabidopsis and rice. Plant Physiol. 140, 411-432. doi: 10.1104/pp.105.073783

Ning, S., Wang, N., Sakuma, S., Pourkheirandish, M., Koba, T., and Komatsuda, T. (2013). Variation in the wheat AP2 homoeologs, the genes underlying lodicule development. Breeding Sci. 63, 255-266. doi: 10.1270/jsbbs.63.255

Nole-Wilson, S., Tranby, T. L., and Krizek, B. A. (2005). AINTEGUMENTA-like (AIL) genes are expressed in young tissues and may specify meristematic or division-competent states. Plant Mol. Biol. 57, 613-628. doi: 10.1007/ s11103-005-0955-6

Okamuro, J. K., Caster, B., Villarroel, R., Van Montagu, M., and Jofuku, K. D. (1997). The AP2 domain of APETALA2 defines a large new family of DNA binding proteins in Arabidopsis. Proc. Natl. Acad. Sci. U. S. A. 94, 7076-7081. doi: 10.1073/pnas.94.13.7076

Pearce, S., Vazquez-Gross, H., Herin, S. Y., Hane, D., Wang, Y., Gu, Y. Q., et al. (2015). WheatExp: an RNA-seq expression database for polyploid wheat. BMC Plant Biol. 15, 299. doi: 10.1186/s12870-015-0692-1

Rashid, M., Guangyuan, H., Guangxiao, Y., Hussain, J., and Xu, Y. (2012). AP2/ERF transcription factor in rice: genome-wide canvas and syntenic relationships between Monocots and Eudicots. Evol. Bioinform. Online 8, 321-355. doi: 10.4137/EBO.S9369

Sakuma, Y., Liu, Q., Dubouzet, J. G., Abe, H., Shinozaki, K., and YamaguchiShinozaki, K. (2002). DNA-binding specificity of the ERF/AP2 domain of Arabidopsis DREBs, transcription factors involved in dehydration- and coldinducible gene expression. Biochem. Biophys. Res. Commun. 290, 998-1009. doi: 10.1006/bbrc.2001.6299

Scheres, B., and Krizek, B. A. (2018). Coordination of growth in root and shoot apices by AIL/PLT transcription factors. Curr. Opin. Plant Biol. 41, 95-101. doi: 10.1016/j.pbi.2017.10.002

Shigyo, M., Hasebe, M., and Ito, M. (2006). Molecular evolution of the AP2 subfamily. Gene 366, 256-265. doi: 10.1016/j.gene.2005.08.009

Simons, K. J., Fellers, J. P., Trick, H. N., Zhang, Z. C., Tai, Y. S., Gill, B. S., et al. (2006). Molecular characterization of the major wheat domestication gene Q. Genetics 172, 547-555. doi: 10.1534/genetics.105.044727

Tamura, K., Stecher, G., Peterson, D., Filipski, A., and Kumar, S. (2013). MEGA6: Molecular Evolutionary Genetics Analysis version 6.0. Mol. Biol. Evol. 30, 2725-2729. doi: 10.1093/molbev/mst197

Tang, M., Li, G., and Chen, M. (2007). The phylogeny and expression pattern of APETALA2-like genes in rice. J. Genet. Genomics. 34, 930-938. doi: 10.1016/ S1673-8527(07)60104-0

Wang, J., Lin, Z., Zhang, X., Liu, H., Zhou, L., Zhong, S., et al. (2019a). krn1, a major quantitative trait locus for kernel row number in maize. New Phytol. 223, 1634-1646. doi: 10.1111/nph.15890
Wang, T., Ping, X., Cao, Y., Jian, H., Gao, Y., Wang, J., et al. (2019b). Genomewide exploration and characterization of miR172/euAP2 genes in Brassica napus L. for likely role in flower organ development. BMC Plant Biol. 19, 336. doi: 10.1186/s12870-019-1936-2

Wang, P., Cheng, T., Lu, M., Liu, G., Li, M., Shi, J., et al. (2016a). Expansion and functional divergence of AP2 group genes in spermatophytes determined by molecular evolution and Arabidopsis mutant analysis. Front. Plant Sci. 7, 1383. doi: 10.3389/fpls.2016.01383

Wang, M., Yue, H., Feng, K., Deng, P., Song, W., and Nie, X. (2016b). Genomewide identification, phylogeny and expressional profiles of mitogen activated protein kinase kinase kinase (MAPKKK) gene family in bread wheat (Triticum aestivum L.). BMC Genomics 17, 668. doi: 10.1186/s12864-016-2993-7

Xie, Q., Li, N., Yang, Y., Lv, Y., Yao, H., Wei, R., et al. (2018). Pleiotropic effects of the wheat domestication gene $Q$ on yield and grain morphology. Planta 247, 1089-1098. doi: 10.1007/s00425-018-2847-4

Xu, B. J., Chen, Q., Zheng, T., Jiang, Y. F., Qiao, Y. Y., Guo, Z. R., et al. (2018). An overexpressed $Q$ allele leads to increased spike density and improved processing quality in common wheat (Triticum aestivum). G3 (Bethesda) 8, 771-778. doi: 10.1534/g3.117.300562

Xu, Z. S., Chen, M., Li, L. C., and Ma, Y. Z. (2011). Functions and application of the AP2/ERF transcription factor family in crop improvement. J. Integr. Plant Biol. 53, 570-585. doi: 10.1111/j.1744-7909.2011.01062.x

Yant, L., Mathieu, J., Dinh, T. T., Ott, F., Lanz, C., Wollmann, H., et al. (2010). Orchestration of the floral transition and floral development in Arabidopsis by the bifunctional transcription factor APETALA2. Plant Cell 22, 2156-2170. doi: $10.1105 /$ tpc. 110.075606

Zhang, K., Han, Y. T., Zhao, F. L., Hu, Y., Gao, Y. R., Ma, Y. F., et al. (2015a). Genome-wide identification and expression analysis of the CDPK gene family in grape, Vitis spp. BMC Plant Biol. 15, 164. doi: 10.1186/s12870-015-0552-Z

Zhang, B., Wang, L., Zeng, L., Zhang, C., and Ma, H. (2015b). Arabidopsis TOE proteins convey a photoperiodic signal to antagonize CONSTANS and regulate flowering time. Genes Dev. 29, 975-987. doi: 10.1101/gad.251520.114

Zhang, J. Z. (2003). Evolution by gene duplication: an update. Trends Ecol. Evol. 18, 292-298. doi: 10.1016/S0169-5347(03)00033-8

Zhang, Z., Belcram, H., Gornicki, P., Charles, M., Just, J., Huneau, C., et al. (2011). Duplication and partitioning in evolution and function of homoeologous $Q$ loci governing domestication characters in polyploid wheat. Proc. Natl. Acad. Sci. U. S. A. 108, 18737-18742. doi: 10.1073/pnas.1110552108

Zhao, Y., Cheng, X., Liu, X., Wu, H., Bi, H., and Xu, H. (2018). The wheat MYB transcription factor TaMYB31 is involved in drought stress responses in Arabidopsis. Front. Plant Sci. 9, 1426. doi: 10.3389/fpls.2018.01426

Zhao, Y., Tian, X., Li, Y., Zhang, L., Guan, P., Kou, X., et al. (2017). Molecular and functional characterization of wheat ARGOS genes influencing plant growth and stress tolerance. Front. Plant Sci. 8, 170. doi: 10.3389/fpls.2017.00170

Conflict of Interest: The authors declare that the research was conducted in the absence of any commercial or financial relationships that could be construed as a potential conflict of interest.

Copyright (c) 2019 Zhao, Ma, Xu, Bi, Xia and Peng. This is an open-access article distributed under the terms of the Creative Commons Attribution License (CC BY). The use, distribution or reproduction in other forums is permitted, provided the original author(s) and the copyright owner(s) are credited and that the original publication in this journal is cited, in accordance with accepted academic practice. No use, distribution or reproduction is permitted which does not comply with these terms. 